\title{
Influence of Rho kinase inhibitor fasudil on late endothelial progenitor cells in peripheral blood of COPD patients with pulmonary artery hypertension
}

\author{
Liu $\mathrm{P}^{1}$, Zhang $\mathrm{HM}^{2}$, Tang $\mathrm{YJ}^{2}$, Sheng $\mathrm{CF}^{1}$, Liu $\mathrm{JX}^{3}$, Zeng $\mathrm{YJ}^{4}$ \\ Department of Respiratoy Diseases, Taihe Hospital of Hubei Medical University, Shiyan, Hubei, PR China. \\ houyu791614@sina.com
}

\begin{abstract}
Objective: To investigate the influence of Fasudil, a Rho inhibitor on the number and functions of the late endothelial progenitor cells in peripheral blood of chronic obstructive pulmonary diseases (COPD) patients with pulmonary artery hypertension.

Background: It is not clear yet, whether Rho Kinase Inhibitor Fasudil can reduced pulmonary artery pressure through improving lung endothelial function.

Methods: 80 COPD patients with pulmonary artery hypertension were selected and divided into two groups: the treatment group and the control group, which had 40 patients, respectively. Changes in the number and function of the late endothelial progenitor cells in peripheral blood of the patients before and after the treatment were compared between the two groups. The changes on the pulmonary artery pressure were also compared. Results: The number of the late endothelial progenitor cells in peripheral blood of the treatment group increased and the function was enhanced. The pulmonary artery pressure was reduced. The difference before and after the treatment and with the control group was statistically significant $(p<0.05)$.

Conclusions: The Rho-kinase inhibitor Fasudil increased the number and enhanced the function of the late endothelial progenitor cells in peripheral blood of COPD patients with pulmonary artery hypertension (Tab. 3, Fig. 2, Ref. 17). Text in PDF www.elis.sk.

Key words: Rho kinase inhibitor Fasudil, chronic obstructive pulmonary diseases, pulmonary artery hypertension, endothelial progenitor cells.
\end{abstract}

Hypoxic pulmonary artery hypertension $(\mathrm{PAH})$ is the core stage of the pathogenetic process of chronic obstructive pulmonary diseases (COPD). The decrease of the pulmonary artery pressure retards the pathogenesis and development of COPD significantly (1). The search for the ideal method to decrease the pulmonary artery pressure is the key to prevent and cure COPD and cor pulmonale. Studies in recent years demonstrated that the damage and malfunction of endothelium play an important role in the pathogenesis and development of PAH (2). The endothelial progenitor cells (EPC) participate not only in the formation of human embryonic vessels, but also in the neogenesis of blood vessels after birth and the repair after endothelium damage (3). Korean scientists Hur et al (4) reported in 2004 the presence of EPC of two kinds of different biological characteristics in adult peripheral blood and named these two kinds of peripheral blood EPC, early EPC and late EPC. Following research considered the late EPC, coming from the bone marrow, as the real EPC, which directly takes part

${ }^{1}$ Intensive Care Unit, Taihe Hospital of Hubei Medical University, Shiyan, Hubei, PR China, ${ }^{2}$ Department of Respiratoy Diseases, Taihe Hospital of Hubei Medical University, Shiyan, Hubei, PR China, ${ }^{3}$ Department of Ultrasound Imaging, Taihe Hospital of Hubei Medical University, Shiyan, Hubei, PR China, and ${ }^{4}$ Biomechanics and Medical Information Institute, Beijing University of Technology, Beijing, PR China

Address for correspondence: H.M. Zhang, Department of Respiratoy Diseases, Taihe Hospital of Hubei Medical University, Shiyan, Hubei, 442000, PR China. Phone: 18772817340 in the repair of vascular endothelium and plays an important role in the neogenesis and repair of blood vessels (5). In recent years, the relationship between Rho/Rho-kinase signaling pathway and cardiopulmonary vascular diseases has drawn an increasing attention. This research investigated the influence of Fasudil, a Rho kinase inhibitor on the number and functions of the late endothelial progenitor cells in peripheral blood of COPD patients with pulmonary artery hypertension by the intervention of Rho kinase inhibitor Fasudil in COPD patients with pulmonary artery hypertension.

\section{Materials and methods}

\section{Research objects}

A total of 80 COPD patients with pulmonary artery hypertension hospitalized from July 2010 to April 2012 were selected, male patients represented 40 cases and female 40 cases. The age of the patients ranged from 50 to 75 years old. The average age was $63.5 \pm 11.6$ years, conforming to the diagnosis criteria. The patients with diabetes, liver and kidney disease, blood diseases, recent surgery, wounds, inflammation, tumor, cerebrovascular disorders and acute myocardial infarction that could interfere with the EPC were excluded. The selected patients did not receive statins. The patients were divided randomly into two groups. The treatment group had 40 patients, out of them were 22 male and 18 female. The control group also had 40 patients, 24 male and 16 female. The age and sex differences between the two groups 
were not statistically different. All the research objects had been informed in detail about the research process. Prior to taking part in the research the patients have given informed consent and the study was approved by the local ethics committee.

\section{Research methods}

All patients of the two groups received routine treatment as oxygen, anti-infectious treatment and phlegm dissolving. The treatment group received intravenous drip of $30 \mathrm{mg}$ of Fasudil hydrochloride inj. with $100 \mathrm{ml}$ physiological saline added by. It was applied twice a day and 21 days was the treatment period.

\section{Measurement of pulmonary artery pressure}

The 5500 type color Doppler ultrasound of HP was used. Round chamber view section of the ventricular apex was selected to observe the regurgitation of the tricuspid valve and transprosthetic gradient $(\Delta \mathrm{P})$. It was estimated that the systolic pressure of pulmonary artery was $\Delta \mathrm{P}+10 \mathrm{mmHg}$ (right atrial pressure).

\section{Separation, culture and identification of late EPC}

The peripheral vein blood of the subjects was collected and the mononuclear cells were separated by density gradient centrifugal method. The obtained mononuclear cell suspension was inoculated to a 24 pore cell culture plate covered by human fibronectin. The inoculation density was $2 \times 10^{6}$ cells $/ \mathrm{ml}$. The plate was cultured in an incubator of $\mathrm{CO}_{2}$ at $37{ }^{\circ} \mathrm{C}$. The fluid was changed for the first time after 4 days. Then, it was changed every 2 days until the 21 st day of culture. The cells were washed with PBS for further analysis. The adherent cells were collected to measure the positivity of cell phenotypes CD34 and KDR with a flow cytometry and the absorption of DiLDL combined with FITC-UEA-I (double positivity) by the laser scanning confocal microscope.

\section{Late EPC count}

The obtained late EPC were counted under 200 times inverted microscope and the average was obtained. The cellular morphology of early and late EPC, respectively was observed.

\section{Measurement of the proliferation ability of late EPC}

The adherent cells in the primary culture were digested by $0.25 \%$ trypsinase and prepared to be single cell suspension with culture fluid. The cell suspension concentration was adjusted to $3^{*} 10^{5} / \mathrm{mL}$. It was inoculated to a 96 pore plate with the density of 104 for every pore. After $48 \mathrm{~h}$ of culture, its reproduction ability was measured by MTT method.

\section{Measurement of adherence ability}

The adherent cells were digested by $0.25 \%$ trypsinase and suspended in $500 \mu \mathrm{L}$ culture fluid for counting. Then the equal number of EPC was spread on a culture plate covered by HFN and cultured for $30 \mathrm{~min}$ at $37{ }^{\circ} \mathrm{C}$. Then the adherent cells were counted under 200 time microscope.

\section{Measurement of migration ability}

The adherent cells were collected and counted as above. 25
Tab. 1. Comparison of age, sex, blood glucose, blood pressure, total cholesterol and low density lipoprotein (LDL) between the two groups.

\begin{tabular}{lcc}
\hline Clinical information & Treatment group & Control group \\
\hline Age (years old) & $61.3 \pm 5.5$ & $63.7 \pm 7.6$ \\
Sex (male/female ratio) & $22: 18$ & $24: 16$ \\
Smoking (case) & $16(40 \%)$ & $18(45 \%)$ \\
Hypertension (case) & $4(10 \%)$ & $6(15 \%)$ \\
Blood glucose (mmol/l) & $5.27 \pm 1.17$ & $4.83 \pm 1.03$ \\
Total cholesterol (mmol/l) & $4.71 \pm 1.45$ & $4.15 \pm 1.47$ \\
Low density lipoprotein $(\mathrm{mmol} / \mathrm{l})$ & $2.93 \pm 0.94$ & $2.75 \pm 0.90$ \\
\hline
\end{tabular}

The differences in age, sex, blood glucose, blood pressure, total cholesterol and low density lipoprotein between the two groups were not statistically significant.

$\mu \mathrm{L}$ culture fluid and vascular endothelial growth factor (VEGF, 50 $\mu \mathrm{g} / \mathrm{L}$ ) were added to the lower chamber of the improved Boyden chambers. $2 * 104$ EPC suspended in $50 \mu \mathrm{L}$ culture fluid was added to the upper chamber. When cultured for $24 \mathrm{~h}$, the unmigrated cells at the surface of the filter membrane were removed and fixated by methane and then stained by Giemsa. 3 random microscope fields (x 400) were selected to count the cells migrated to the bottom.

\section{Statistics}

Statistical analysis was conducted by SPSS13. 0 statistical software. The data was demonstrated by $\mathrm{x} \pm \mathrm{s}$. The comparison between mean values was conducted with analysis of variance and the comparison between rates was conducted with $\chi 2$ test. Using $p$ $<0.05$ as the variance was considered as statistically significant.

\section{Results}

\section{Basic information}

Comparison of age, sex, blood glucose, blood pressure, total cholesterol and low density lipoprotein (LDL) between the two groups. The differences in age, sex, blood glucose, blood pressure, total cholesterol and low density lipoprotein between the two groups were not statistically significant (Tab. 1).

\section{Late EPC identification}

The late EPC cultured in isolation was measured for the positivity of cell phenotypes of CD34 and KDR by a flow cytometry (Fig. 1) and the absorption of DiLDL combined with FITC-UEA-I (double positivity) (Fig. 2).

\section{Comparison of systolic pressure of pulmonary artery before and after treatment of two groups}

In the treatment group the pulmonary artery pressure was reduced and the ray fraction was increased. The difference before and after the treatment and with the control group was statistically significant $(\mathrm{p}<0.05)$ (Tab. 2).

Changes in the number and function of late EPC in peripheral blood before and after treatment of the two groups

The number of the late endothelial progenitor cells in peripheral blood of the treatment group increased and the function was enhanced. The difference before and after the treatment and with the control group was statistically significant $(\mathrm{p}<0.05)$. The changes in the number and function of the late endothelial progeni- 


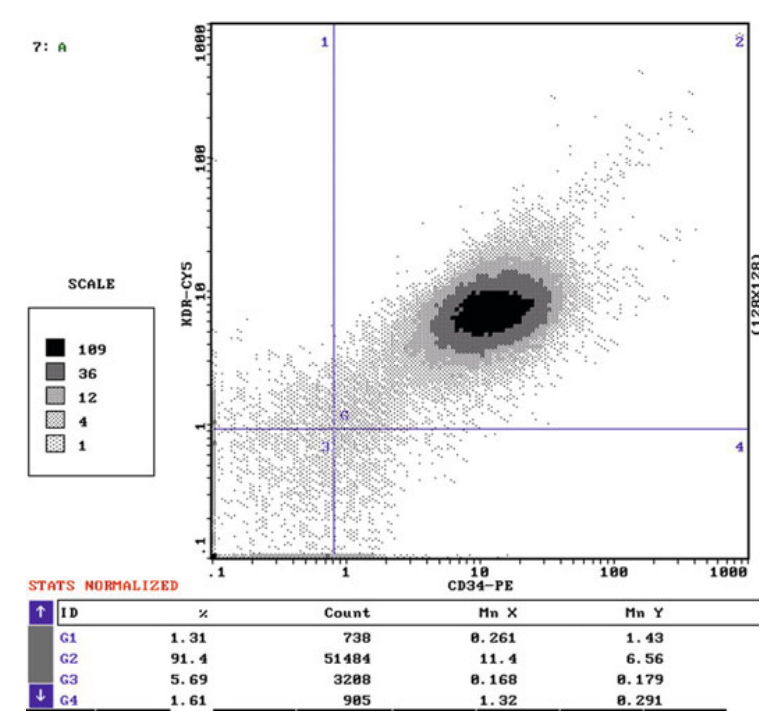

Fig. 1. The late EPC cultured in isolation was measured for the positivity of cell phenotypes of CD34 and KDR by a flow cytometry.

tor cells in peripheral blood before and after the treatment of the control group were not statistically significant $(p>0.05)$ (Tab. 3).

\section{Discussion}

The reformation of pulmonary vessels is the main factor for the pathogenesis of COPD with pulmonary artery hypertension. It is generally believed that systematic endothelial damage is the starting phase of pulmonary artery hypertension. The damage and / or malfunction of pulmonary endothelial cells might cause endogenous unbalance between pulmonary vasodilator and vasoconstrictor. Thus, the relaxation vascular active substance decreases and the contracting vascular active substance increases (6). In addition, the damaged endothelial cells secrete multiple cell factors, such as fibroblast growth factor, platelet derived growth factor, etc. to promote hyperplasia and hypertrophy of vascular smooth muscle cells. In a pulmonary artery hypertension case, the damage of vascular endothelial cells is one of the factors in the pathogenetic

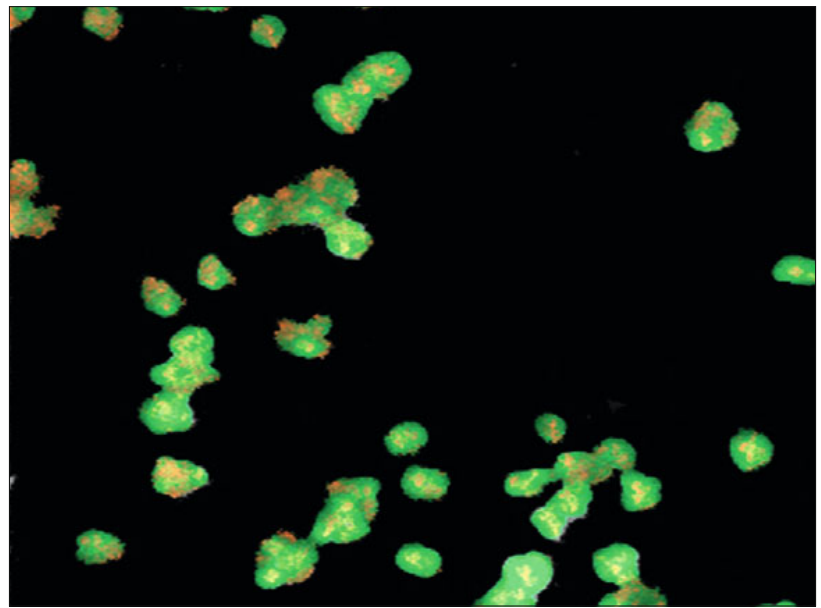

Fig. 2. The absorption of DiLDL combined with FITC-UEA-I (double positivity).

processes. If this process is inhibited, the development of pulmonary artery hypertension could be possibly inhibited. EPC is reproductive and could be differentiated to be vascular endothelial cells to accelerate the repair of damaged vascular endothelium. Studies showed that endogenous erythrocyte stimulating factor was able to promote the EPC mobilization from chronic hypoxia mouse bone marrow to prevent the $\mathrm{PAH}$ process and reduce the hypertrophy of the right ventricle and the reconstruction of pulmonary vessels $(7,8)$. As EPC has an important role in maintaining the complete functions of endothelium, the treatment of PAH by EPC also draws the attention of researchers home and abroad.

This research found that the pulmonary artery pressure of the patients in the Fasudil treatment group was reduced significantly. The number and function of the late endothelial progenitor cells in peripheral blood were also enhanced. It is conjectured that the following mechanisms are possible (1). In recent years, the relationship between Rho/Rho-kinase signaling pathway and cardiopulmonary vascular diseases has drawn an increasing attention. Fasudil is a selective inhibitor of Rho-kinase signaling pathway. It competes for the combination with the ATO combination site in the kinase area of Rho-kinase with ATP, so as to block the activity

Tab. 2. Comparison of systolic pressure of pulmonary artery before and after treatment between the two groups.

\begin{tabular}{llcc}
\hline & & $\begin{array}{c}\text { Systolic pressure of pulmonary } \\
\text { artery }(\mathrm{mmHg})\end{array}$ & $\begin{array}{l}\text { Internal diameter of outflow } \\
\text { tract of right ventricle }(\mathrm{mm})\end{array}$ \\
\hline $\begin{array}{l}\text { Treatment group } \\
\mathrm{n}=40\end{array}$ & before treatment & $49.00 \pm 4.64$ & $33.10 \pm 3.53$ \\
\cline { 2 - 4 } $\begin{array}{l}\text { Control group } \\
\mathrm{n}=40\end{array}$ & after treatment & $25.00 \pm 3.26^{1}$ & $27.50 \pm 1.26^{1}$ \\
\hline
\end{tabular}

${ }^{1}$ Variance is statistically different compared to the value before treatment, $\mathrm{p}<0.05 ;{ }^{2}$ Variance is statistically different compared to the treatment group, $\mathrm{p}<0.05$.

Tab. 3. Changes in the number and function of late EPC in peripheral blood before and after treatment of the two groups.

\begin{tabular}{|c|c|c|c|c|c|}
\hline & & Number & reproduction ability & migration ability & adherence ability \\
\hline \multirow{2}{*}{$\begin{array}{l}\text { Treatment group } \\
n=40\end{array}$} & before treatment & $14.35 \pm 6.32$ & $0.15 \pm 4.55$ & $9.10 \pm 4.81$ & $10.50 \pm 1.28$ \\
\hline & after treatment & $30.12 \pm 2.26^{1}$ & $0.29 \pm 2.25^{1}$ & $17.00 \pm 3.27^{1}$ & $24.85 \pm 2.32^{1}$ \\
\hline \multirow{2}{*}{$\begin{array}{l}\text { Control group } \\
n=40\end{array}$} & before treatment & $14.58 \pm 3.51$ & $0.15 \pm 2.44$ & $9.05 \pm 1.27$ & $10.28 \pm 2.37$ \\
\hline & after treatment & $16.15 \pm 2.74^{2}$ & $0.14 \pm 3.29^{2}$ & $10.00 \pm 2.19^{2}$ & $10.58 \pm 2.56^{2}$ \\
\hline
\end{tabular}

${ }^{1}$ Variance is statistically different compared with the value before treatment, $\mathrm{p}<0.05 ;{ }^{2}$ Variance is statistically different compared with the treatment group, $\mathrm{p}<0.05$. 
of Rho-kinase. It could also inhibit multiple proteinases such as myosin light chain proteinase and protein kinase, leading to the inhibition of phosphorylation at the final stage of contraction of smooth muscle. Thus, the vessels are expanded (9). Fagan et al (10) found that the selective inhibitors -Y-27632 and Fasudil of Rho/Rho-kinase signaling pathway were able to lower the pulmonary artery pressure and pulmonary vascular resistance in mice with pulmonary artery hypertension and had important adjustment effects on the vascular structure and function (11). Recent studies proved that Fasudil could inhibit the synthesis and secretion of ET-1 by endothelial cells and promote the synthesis and secretion of NO, to improve the balance between ET-1 and NO. Therefore, the endothelium mediated relaxation vascular effect was enhanced (12). Therefore, Fasudil could enlarge the vessels by Rho/Rho-kinase signaling pathway to reduce the pulmonary artery pressure; furthermore, it could adjust the endothelial cells function so that the latter decreases the secretion of blood vessel contraction substance and increases relaxation substances. In this way, the pulmonary artery pressure is lowered (2). With the development of COPD, the peripheral airway obstruction, lung parenchyma damage and abnormality of pulmonary blood vessels reduce the pulmonary gas exchange capacity and causes hyoxemia, even hypercapnia. Long lasting chronic hypoxia results in pulmonary artery hypertension. The main mechanism is hypoxic pulmonary vasoconstriction (HPV), dysfunction of endothelium of pulmonary vessels and reconstruction of pulmonary vessels. The vascular endothelial cells have important role in adjusting human pulmonary circulation. The endothelial cells could release multiple vascular relaxation substances to adjust the vascular reactions. These substances are called endothelium derived relaxation factors (EDRF) (13). N0 is one of them. In COPD patients with pulmonary artery hypertension, EDRF mediated vascular contraction is damaged, pulmonary vascular resistance (RPV) is increased. These are the results of endothelial damage caused by shear stress of hemodynamics and hypoxia (14). The endothelial progenitor cells (EPCs) are precursor cells that can be differentiated into vascular endothelial cells directly. EPC plays an important role in the neogenesis and maintaining of the completeness of the function of endothelium of blood vessels (15). Yamada et al (16) proved that stem cells were necessary for the repair of pulmonary tissues with the lipopolysaccharide mediated mouse pneumonia model. Nagaya et al. (17) reported that in the monocrotaline mediated pulmonary artery hypertension nude mouse model, xenotransplantation of EPC reduced the pulmonary vascular resistance by $16 \%$. Gene infected EPC could reduce the pulmonary vascular resistance by $35 \%$ and its survival rate was higher (17). These studies confirmed that EPC could enhance the damaged pulmonary artery endothelial repair and the reconstruction of artery, so as to reduce the pulmonary artery pressure.

In this research, Fasudil reduced the pulmonary artery pressure of COPD patients with pulmonary artery hypertension. One of the mechanisms is the increase in the number and function of the late EPC in peripheral blood of the patients to reduce the damage of pulmonary vascular endothelial cells and improve the reconstruction of pulmonary vascular structure, so as to obtain the treatment effects.

\section{References}

1. Melek M, Esen O, Esen A M et al. Tissue Doppler evaluation of tricuspid annulus for estimation of pulmonary artery pressure in patients with COPD. J Lung 2006; 184 (3): 121-131.

2. Yue WS, Wang M, Yan GH et al. Smoking is associated with depletion of circulating endothelial progenitor cells and elevated pulmonary artery systolic pressure in patients with coronary artery disease. J Am J Cardiol 2010; 106 (9): 1248-1254.

3. Kwon SM, Lee YK, Yokoyama A et al. Differential activity of bone marrow hematopoietic stem cell subpopulations for EPC development and ischemic neovascularization. J Mol Cell Cardiol 2011; 51 (3): 308-317.

4. Hur J, Yoon CH, Kim HS et al. Characterization of two types of endothelial progenitor cells and their different contributions to neovasculogenesis. Arterioscler Thromb Vasc Biol 2004; 24 (2): 288-293.

5. Yoon CH, Hur J, Park KW et al. Synergistic neovascularization by mixed transplantation of early endothelial progenitor cells and late outgrowth endothelial cells: the role of angiogenic cytokines and matrix metalloproteinases. Circulation 2005; 112 (11): 1618-1627.

6. Kanazawa H, Asai K, Nomura S. Vascular endothelial growth factor as a non-invasive marker of pulmonary vascular remodeling in patients with bronchitis-type of COPD. J Resp Res 2007; 8: 22.

7. Yang $\mathbf{L}$, Guan $\mathbf{H}, \mathbf{H e} \mathbf{J}$ et al. VEGF increases the proliferative capacity and eNOS/NO levels of endothelial progenitor cells through the calcineurin/ NFAT signalling pathway. J Cell Biol Int 2012; 36 (1): 21-27.

8. Yip HK, Chang LT, Sun CK et al. Autologous transplantation of bone marrow-derived endothelial progenitor cells attenuates monocrotaline-induced pulmonary arterial hypertension in rats. Crit Care Med 2008; 36 (3): 873-880.

9. Ho TJ, Huang CC, Huang CY et al. Fasudil, a Rho-kinase inhibitor, protects against excessive endurance exercise training-induced cardiac hypertrophy, apoptosis and fibrosis in rats. Eur J Appl Physiol 2012; 112 (8): 2943-2955.

10. Fagan KA, Oka M, Bauer NR et al. Attenuation of acute hypoxic pulmonary vasoconstriction and hypoxic pulmonary hypertension in mice by inhibition of Rho-kinase. Am J Physiol Lung Cell Mol Physiol 2004; 287 (4): L656-L664.

11. Mcmurtry IF, Abe K, Ota $\mathbf{H}$ et al. Rho kinase-mediated vasoconstriction in pulmonary hypertension. Adv Exp Med Biol 2010; 661: 299-308.

12. O E, Ahn HY, Kim HK et al. The Rho kinase inhibitor fasudil augments the number of functional endothelial progenitor cells in ex vivo cultures. Int J Mol Med 2011; 28 (3): 357-363.

13. Gryglewski RJ, Moncada S, Palmer RM. Bioassay of prostacyclin and endothelium-derived relaxing factor (EDRF) from porcine aortic endothelial cells. 1985. Br J Pharmacol 1997; 120 (Suppl 4): 494-503, 491-493.

14. Paruchuri S, Yang JH, Aikawa E et al. Human pulmonary valve progenitor cells exhibit endothelial/mesenchymal plasticity in response to vascular endothelial growth factor-A and transforming growth factor-beta2. Circulat Res 2006; 99 (8): 861-869.

15. Dubois C, Liu X, Claus $P$ et al. Differential effects of progenitor cell populations on left ventricular remodeling and myocardial neovascularization after myocardial infarction. J Am Coll Cardiol 2010; 55 (20): 223-243.

16. Yamada M, Kubo H, Kobayashi S et al. Bone marrow-derived progenitor cells are important for lung repair after lipopolysaccharide-induced lung injury. J Immunol 2004; 172 (2): 1266-1272.

17. Nagaya N, Kyotani S, Uematsu $M$ et al. Effects of adrenomedullin inhalation on hemodynamics and exercise capacity in patients with idiopathic pulmonary arterial hypertension. Circulation 2004; 109 (3): 351-356.

Received September 12, 2013. Accepted October 12, 2013. 